\title{
On the reflection of time-domain acoustic spherical waves by a sinusoidal diffraction grating
}

\author{
Guochao $\mathrm{Gao}^{1}$, Paul Cristini ${ }^{2,}$, Nathalie Favretto-Cristini ${ }^{2}$, and Carole Deumié ${ }^{1}$ \\ ${ }^{1}$ Aix-Marseille Univ, CNRS, Centrale Marseille, Institut Fresnel, 13397 Marseille, France \\ ${ }^{2}$ Aix-Marseille Univ, CNRS, Centrale Marseille, LMA, 13453 Marseille, France
}

Received 25 August 2020, Accepted 17 December 2020

\begin{abstract}
This work reports on some results obtained from numerical simulations of time-domain acoustic wave propagation in the presence of a periodically rough interface. Emphasis is put on the structure of the reflected signals in the presence of a sinusoidal grating. More specifically, we investigate the effect of the frequency bandwidth of the emitted signal and the effect of the incident wavefront sphericity on the signals reflected from the rough interface and associated with the different diffraction orders.
\end{abstract}

Keywords: Wave scattering, Periodic rough surface, Grating equation, Numerical simulation, Time domain

\section{Introduction}

The main objective that underlies the work presented here is to better understand the impact of rough interfaces in seismic wave propagation and in seismic imaging, in order to possibly better handle rough interfaces. This is an important topic, as the presence of interfaces with irregularities leads to complex wave propagation phenomena, such as combination of reflections, scattering and other effects, resulting in difficulties in the reconstruction of the shape of the interfaces (useful for both geologists and geophysicists) and in the estimation of seismic parameters (velocity, density, etc). As an example, the rugged topography of the basaltic surfaces generates severe wave distortion and scattering of seismic energy, and hence has a detrimental effect on seismic imaging quality of underlying geological structures $[1,2]$. This detrimental effect is even greater than that provided by internal heterogeneities of basalt layers [3]. As a result, imaging of sub-basalt areas is still a major concern in volcano seismology or in hydrocarbon exploration $[4,5]$. Improvements in this field can then have an impact at different scales, from meters in environmental contexts to kilometers at the exploration scale, and tens of kilometers in a regional scale. Most natural geological topographies, like basalt interfaces, have roughness on scales from centimeters to kilometers, and the roughness pattern appears to be fractal (i.e. identical at different scales) (Martini \& Bean [3]). Nevertheless, in the work presented here, we focus only on periodically rough interfaces, since this kind of interfaces can more easily provide useful physical insights into the

\footnotetext{
*Corresponding author: cristini@lma.cnrs-mrs.fr
}

wave/interface interaction. Moreover, despite elastic effects (e.g. mode conversions, surface waves) play an important role in the real geophysical world, we consider here a very simplified case, namely acoustic waves interacting with sinusoidal interfaces, in order to better understand the effect of roughness characteristics on the spherical wavefronts in the time domain.

Wave reflection from rough interfaces has been widely addressed in literature either in optics, acoustics, or seismics (e.g. [6-10]). In particular, the diffraction of harmonic plane waves by a periodic grating has been deeply investigated, and therefore is now very well known [11, 12]. Indeed, when a monochromatic acoustic wave (with wavelength $\lambda$ ) strikes under the incidence angle $i$ a periodic interface (with a spatial period $d$ ), it gives rise to several diffracted monochromatic waves of order $n$ that propagate along the directions $\theta_{n}$ (Fig. 1), defined by the well-known grating equation [11-14]:

$$
\sin \theta_{n}=\sin i+n \frac{\lambda}{d} n=0, \pm 1, \pm 2, \ldots
$$

In this work, we consider a sinusoidal grating. It is worth noting that this equation is valid for any type of periodic gratings ([14], Appendix D). Considering another type of grating only changes the diffraction efficiency and thus the amplitude of the different diffraction orders.

In the case of an incident non-monochromatic acoustic wave, that carries naturally several wavelengths, the angle $\theta_{n}$ associated with a given diffraction order $n$ is no more unique but lies within a broad range provided by the grating equation. This phenomenon is called angular dispersion. Accordingly, there is also a temporal dispersion 
of the signals associated with the different orders, and the dispersion follows different laws according to the diffraction order. It has to be pointed out here that, as shown by the grating equation, only the higher-order $(|n| \geq 1)$ diffracted waves, and not the specular reflection (i.e. the zeroth-order diffraction), are affected by dispersion.

Replacing plane waves by spherical waves makes the issue of the wave/rough interface interaction much more complex. Indeed, all the plane waves that compose the spherical wave strike the interface under different incidence angles, modifying the condition of existence of a given diffraction order. Very few works have been concerned with the interaction of harmonic acoustic spherical waves with a rough interface (e.g. [15]), and to the best of our knownledge, study of the interaction between a non-monochromatic spherical acoustic wave with a periodic rough interface is still lacking. The main goal of this work is then to analyze the reflection of a non-harmonic spherical wave, propagating in a homogeneous acoustic medium, from a sinusoidal grating. We focus more particularly on the effect of the incident wavefront sphericity on the diffracted waves. The analysis relies on 2D numerical simulations conducted in the time domain.

\section{Configurations and numerical modelling}

The numerical simulations are performed with the open-source package SPECFEM2D ${ }^{1}$ current source [16], published under the CeCILL v2 license, that solves the wave equation without any approximation using a spectralelement method. The Spectral-Element Method (SEM) is based upon a high-order piecewise polynomial approximation of the weak formulation of the wave equation. It combines the accuracy of the pseudospectral method with the flexibility of the finite-element method. In this method, the wavefield is represented in terms of high-degree Lagrange interpolants, and integrals are computed based upon Gauss-Lobatto-Legendre quadrature. This combination leads to perfectly diagonal mass matrix, which in turn leads to a fully explicit time scheme that lends itself very well to numerical simulations on parallel computers. Complex models that include fluid, elastic, viscoelastic, anisotropic or porous media can be modelled, making the SEM a method of choice for the numerical modelling of wave progagation in complex media. Furthermore, the SEM allows for the accurate handling of curved interfaces making this numerical method very well adapted to the solution of the problem of the reflection of a spherical wave from a corrugated surface.

We consider a computational domain with horizontal and vertical sizes of $3000 \mathrm{~m}$ and $1605 \mathrm{~m}$. The domain is homogeneous and composed of a medium with a wave velocity of $1500 \mathrm{~m} / \mathrm{s}$. We consider a point source located at $x=1500 \mathrm{~m}, y=105 \mathrm{~m}$ that emits a tone burst signal with a central frequency $f_{0}$ of $100 \mathrm{~Hz}$ and a bandwidth of $30 \mathrm{~Hz}$. As a consequence, the wavelength at the central frequency

\footnotetext{
${ }^{1}$ https://github.com/geodynamics/specfem $2 \mathrm{~d}$
}

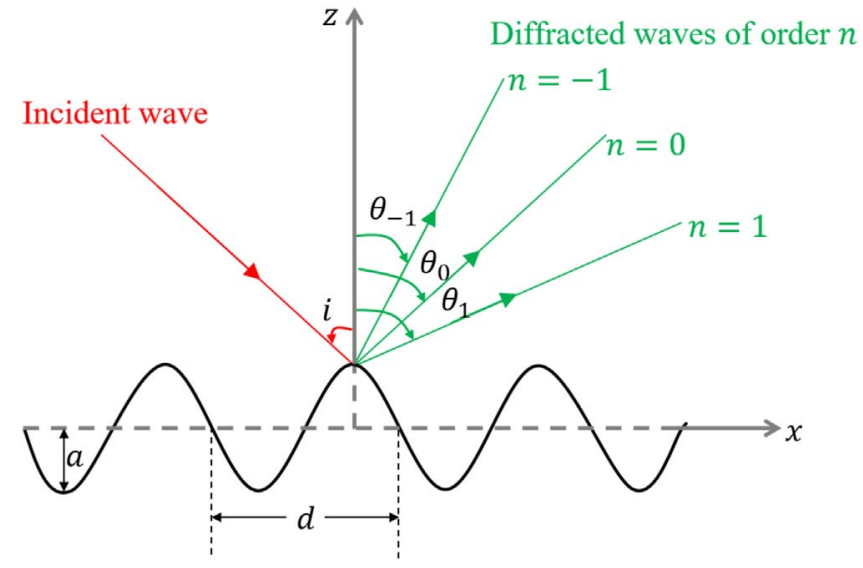

Figure 1. Schematic representation of the reflection of a harmonic plane wave from a periodic rough interface.

$f_{0}$ is $\lambda_{0}=15 \mathrm{~m}$. The bottom is a sine-shaped periodic rough surface whose amplitude and period are $a=\lambda_{0} / 2=7.5 \mathrm{~m}$ and $d=\sqrt{2} \lambda_{0} \approx 21.2 \mathrm{~m}$ respectively. The bottom of the computational model is modelled as a perfectly reflecting surface. The other boundaries are absorbing using perfectly matched layers [17]. In this configuration, the maximum possible value for the incidence angle is $45^{\circ}$.

For the time marching, we use a Newmark scheme which is second-order accurate. The different parameters of the numerical simulations are carefully selected so that we obtain stable and accurate results. As shown in the snapshots presented hereafter, no spurious reflections are observed from the sides of the domain where a perfectly matched layer is implemented.

Because of the complexity of the wavefield generated in this type of configuration, we consider two rough interfaces, namely a half-flat half-rough interface and a fully rough interface, to allow the diffraction orders generated by positive incident angles and orders generated by negative incident angles to be separated. Snapshots of the wavefields after reflection of the incident wavefiled from the rough interfaces are illustrated in Figure 2. It is clearly seen that orders of diffraction greater than two do not propagate in these two configurations. For the half-flat half-rough interface configuration (Fig. 2a), the -2nd-order diffraction is well separated from the \pm 1 st-order diffractions. The \pm 1 st-order diffractions interfere only in the right part of the computational domain. For the fully rough configuration (Fig. 2b), besides the aforementioned diffraction orders, a +2 nd-order diffraction is present. The \pm 2 nd-order diffractions only interfere in an area around the vertical to the source while the \pm 1 st-order diffractions interfere almost everywhere. For the two configurations, spurious diffractions generated by the edges of the rough part of the interfaces are seen (Fig. 2).

The distribution of the diffracted orders, calculated from the grating equation, as a function of the incident angle and the frequency content of the emitted signal is illustrated in Figure 3. For a given diffraction order, the bounds of the domain of existence are given by the curves associated with 


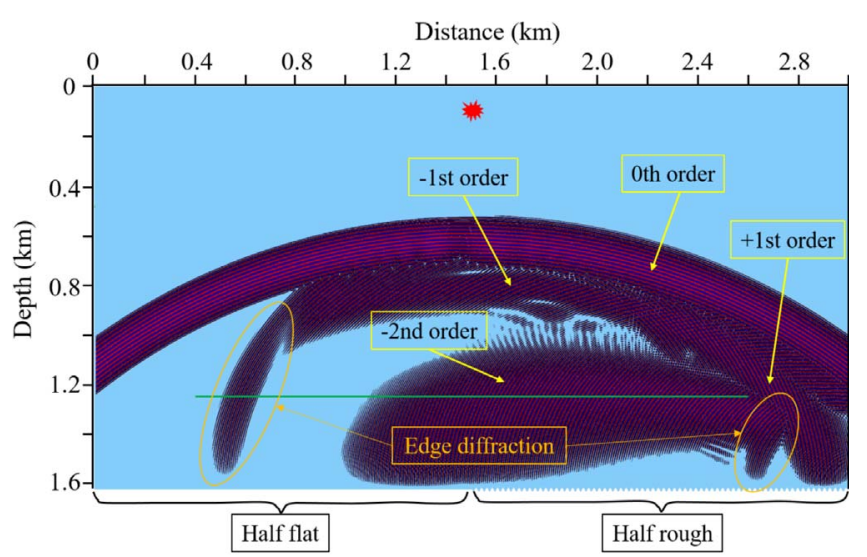

(a) Half-flat half-rough interface.

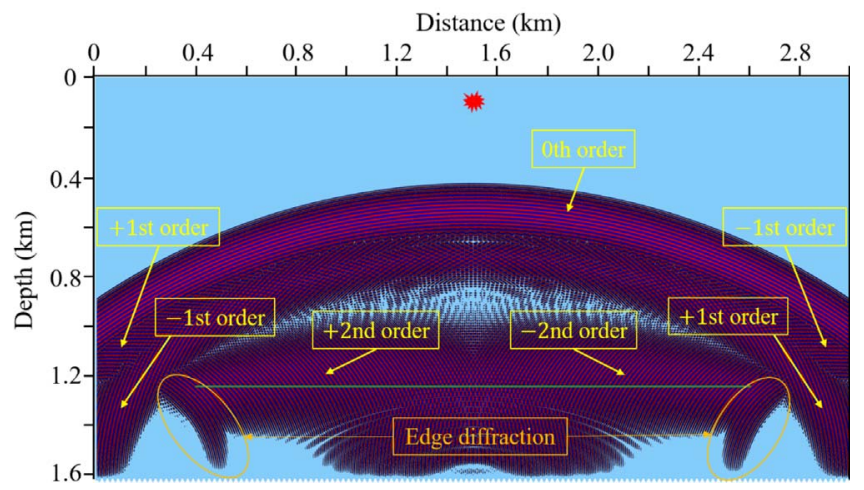

(b) Fully rough interface.

Figure 2. Snapshots (at time (a) $t=1.74 \mathrm{~s}$, (b) $t=1.8 \mathrm{~s}$ ) of the wavefields after reflection of the incident wavefield from (a) a half-flat half-rough interface, (b) a fully rough interface. The source location is indicated by a red star, while the horizontal array of receivers is indicated by a green line.

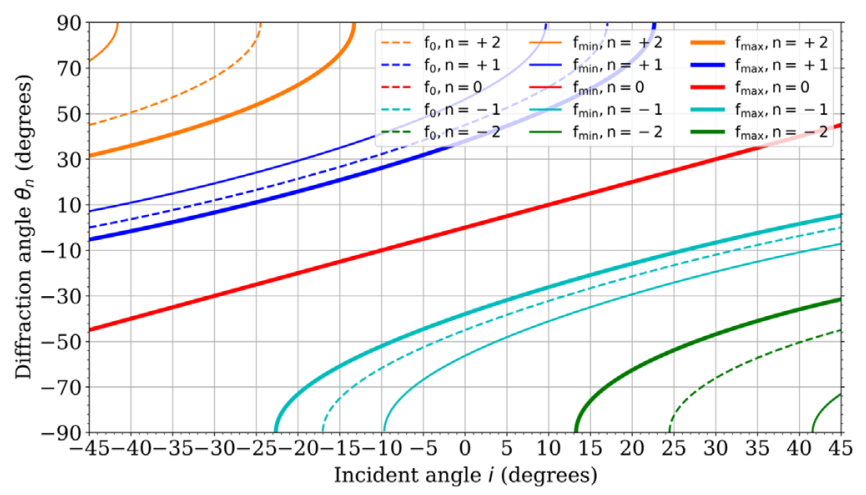

Figure 3. Domains of existence (bounded by full lines) for the different diffracted orders $(0, \pm 1, \pm 2)$ as a function of the incident angle and frequency content of the emitted signal.

the solution of the grating equation for the minimum wavelength (i.e. maximum frequency of the signal) and for the maximum wavelength (i.e. minimum frequency of the signal). From Figure 3, it can be seen that the greater the diffraction order, the wider the domain of its existence, leading to a diffracted signal strongly dispersed.

\section{Time-frequency analysis of the numerical results}

In order to identify the modification of the temporal characteristics of the emitted signal after reflection from the rough interfaces, we perform a time-frequency analysis using a reassigned spectrogram [18]. The use of a reassigned time-frequency representation allows for a more accurate analysis by exhibiting the fine structures of the received signals. Figures $4-6$, present the temporal signal, together with its reassigned spectrogram, recorded at three distinct positions along the horizontal array that is located at a distance of $270 \mathrm{~m}$ from the rough interfaces. For the sake of comparison, the results for the case of a half-flat halfrough interface and for a fully rough interface are shown in Figures 4-6. The first receiver is situated at $x=400 \mathrm{~m}$ (left of the source). Note that this receiver is above the flat part of the half-flat half-rough interface. The second receiver is situated at $x=2300 \mathrm{~m}$ (right of the source) and is above the rough part of the half-flat half-rough interface. The third receiver is situated at the middle of the receiver array at $x=1500 \mathrm{~m}$, so exactly vertical to the source.

The signals received at the first receiver (also noted trace 1) for both the half-flat half-rough and full rough interfaces are shown in Figure 4.

According to Figure 2a, the signal obtained for the case of the half-flat half-rough interface exhibits a first wave packet corresponding to the specular reflection (i.e. the zeroth-order diffraction), and a second wave packet corresponding to diffraction of order -2 (Fig. 4a). In between these two wave packets and after the second wave packet, two small wave packets can be observed corresponding to the diffraction by the edges of the rough interface of finite length. It can be clearly seen that the specular reflection is not affected by the rough interface and that the wave packet associated to the -2 nd diffraction order is strongly dispersed as expected from the analysis of the grating equation. For the case of the fully rough interface, in addition to the wave packet associated to the -2 nd diffraction order, two wave packets associated to the +1 st and -1 st diffraction order are present as well (Fig. 4). These latter packets are much less dispersed than the former one, which is consistent with the results shown in Figure 3.

The signals received at the second receiver, together with its associated reassigned spectrograms, are represented for the two types of interfaces in Figure 5. The 0th-, -1stand -2nd-order diffractions can be clearly seen for the halfflat half-rough configuration (Fig. 5). For the fully rough configuration, in addition to these wave packets, a strongly dispersed wave packet associated with the +2 nd-order diffraction occurs at the end of the signal (Fig. 4). In between, the +2 nd-order wave diffracted packet and the -2nd-order wave diffracted packet, a wave packet of small amplitude and corresponding to the diffraction by the edge of the model can be observed.

The signal received at the third receiver (situated exactly vertical to the source), together with its associated reassigned spectrogram, is shown in Figure 6 for the two types of interfaces. The 0th, -1 st and -2 nd-order 

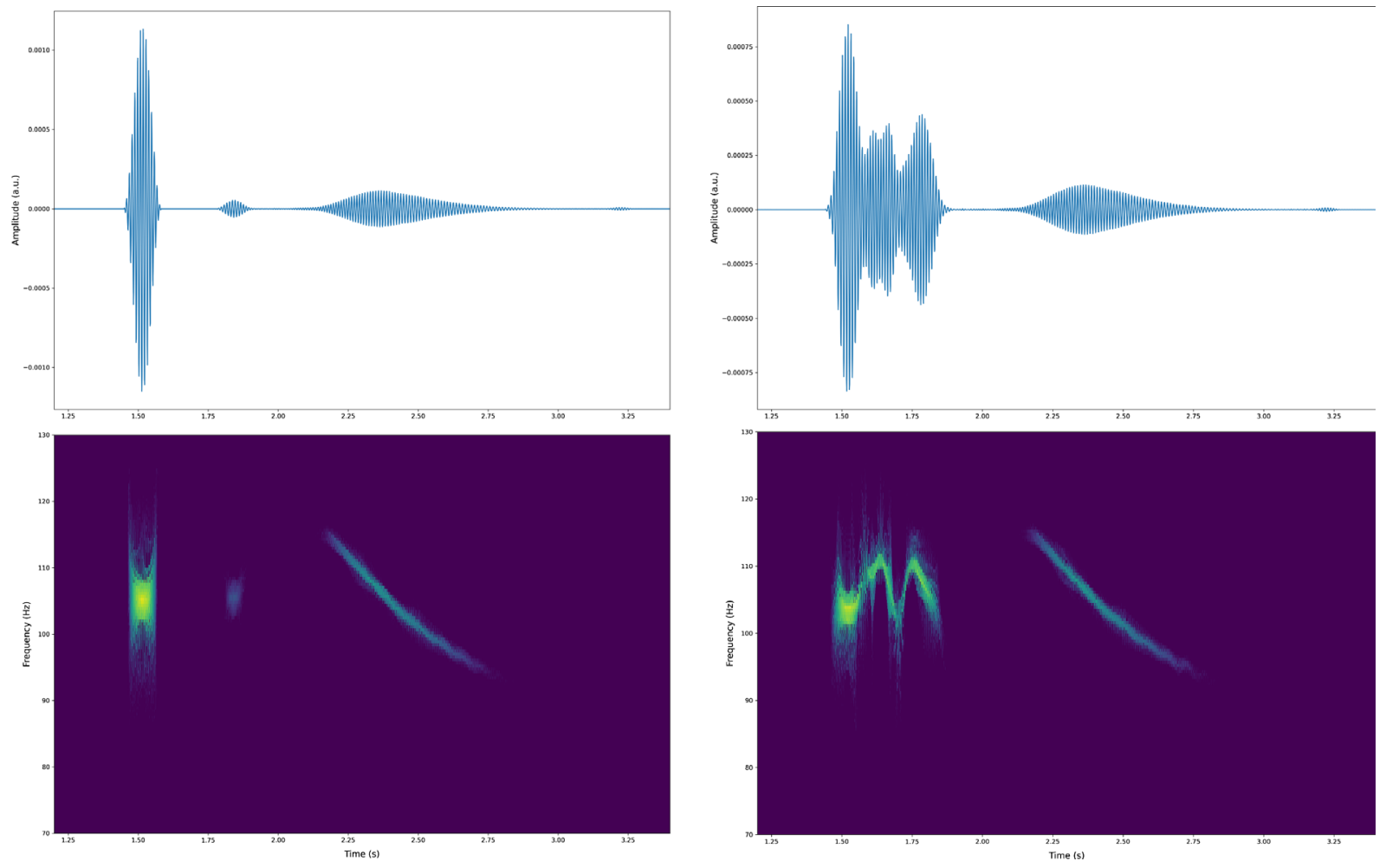

(a) Half-flat half-rough interface.

(b) Fully rough interface.

Figure 4. (Top) Signal (trace 1) received at the first receiver $(x=400 \mathrm{~m})$ and (bottom) associated reassigned spectrogram, for the case of a half-flat half-rough interface (left) and for a fully rough interface (right).
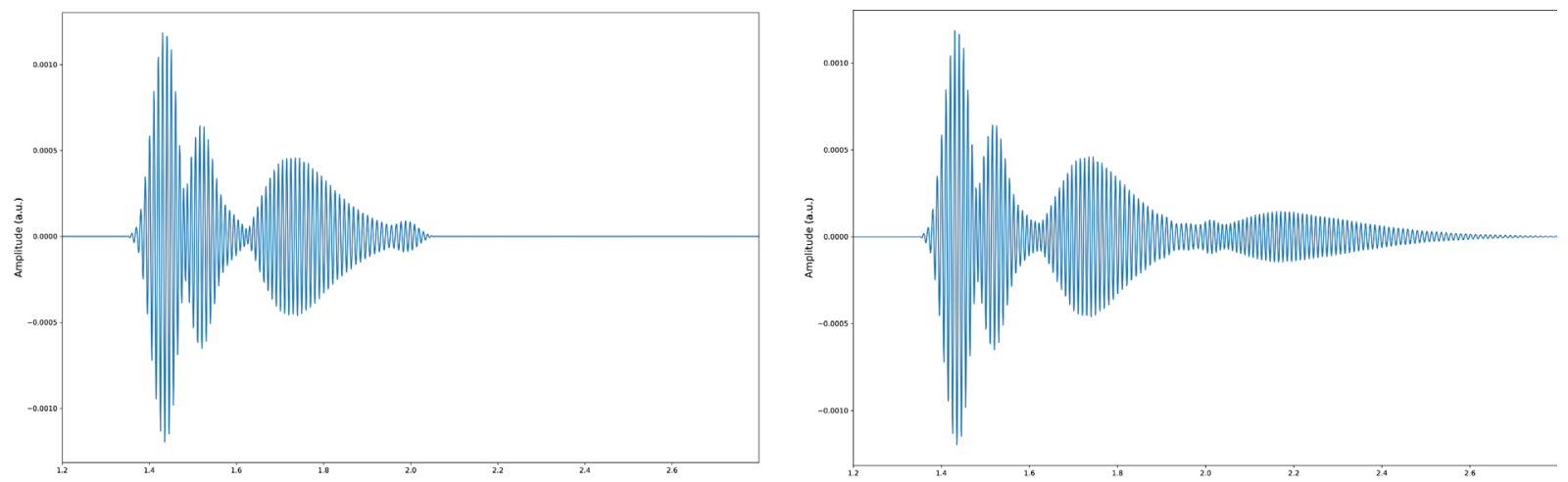

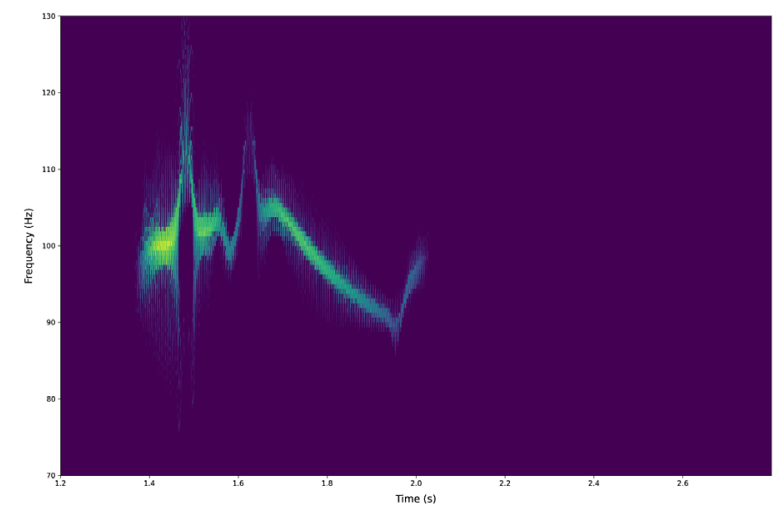

(a) Half-flat half rough interface.

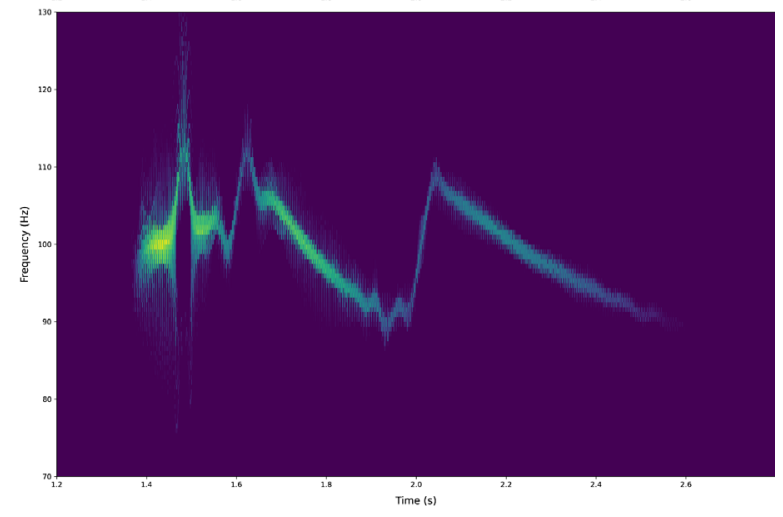

(b) Fully rough interface.

Figure 5. (Top) Signal (trace 475$)$ received at the 475 th receiver $(x=2300 \mathrm{~m})$ and (bottom) associated reassigned spectrogram, for the case of a half-flat half-rough interface (left) and for a fully rough interface (right). 

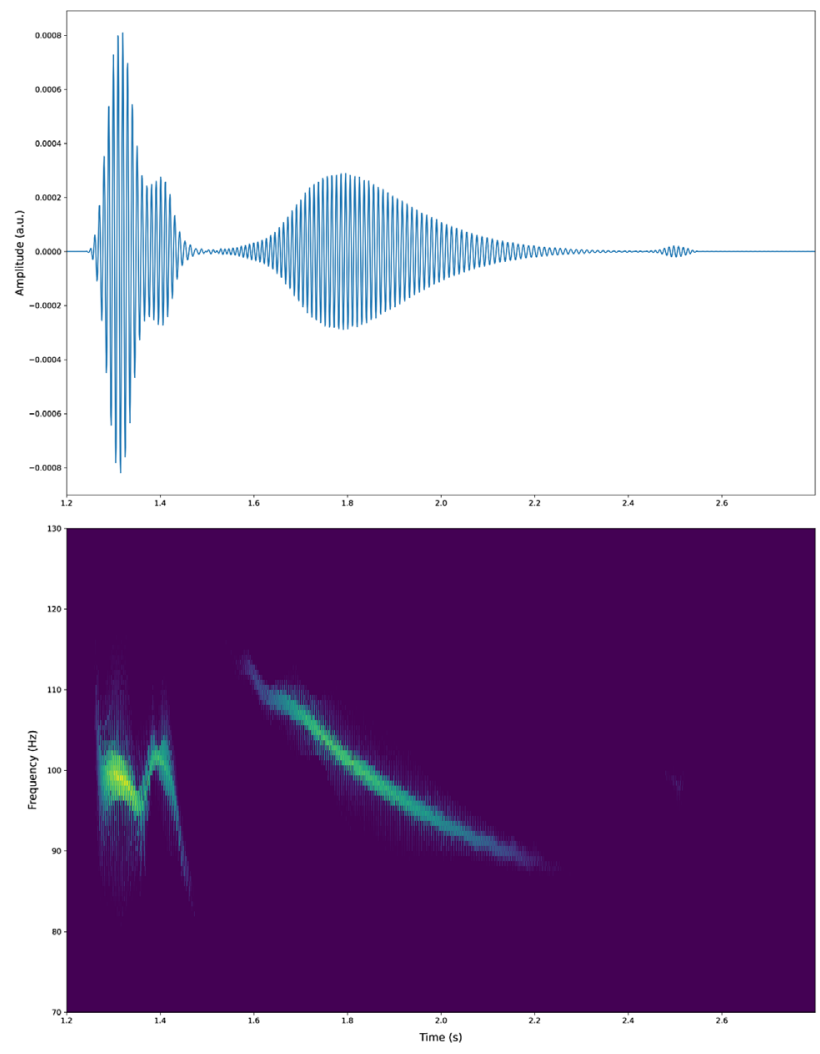

(a) Half-flat half-rough interface.
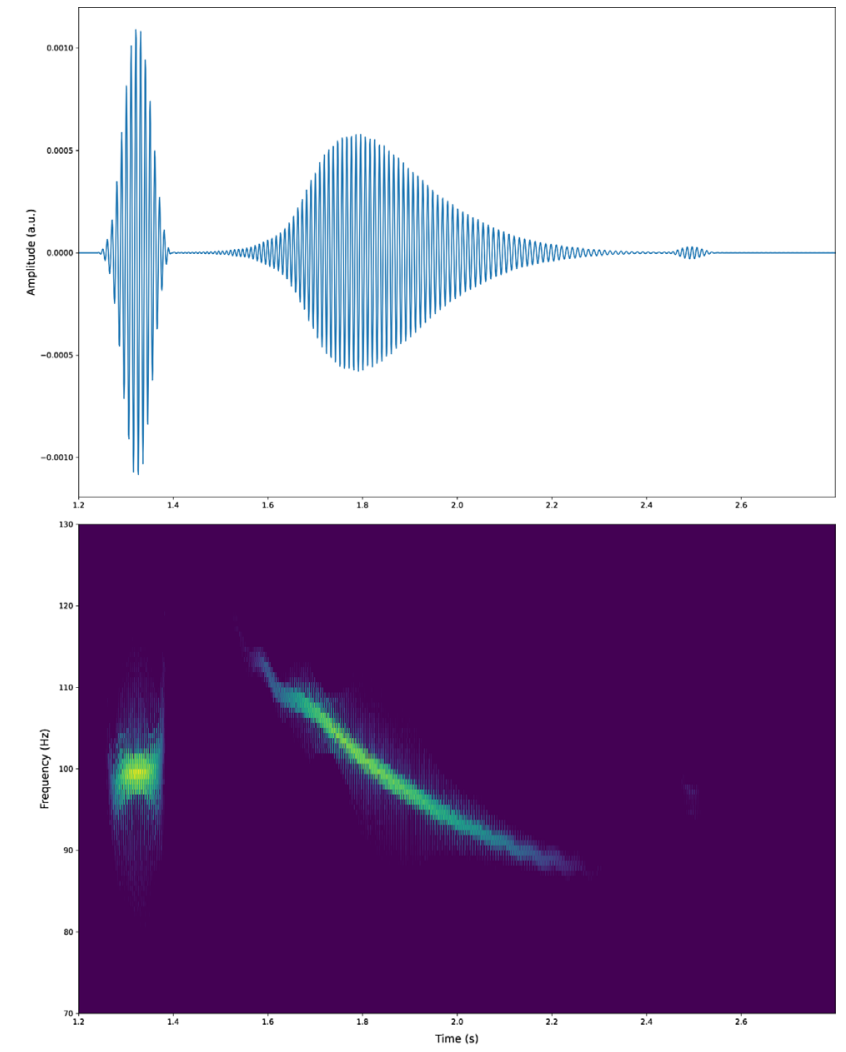

(b) Fully rough interface.

Figure 6. (Top) Signal (trace 276) received at the 276th receiver $(x=1500 \mathrm{~m}$ ) and (bottom) associated reassigned spectrogram, for the case of a half-flat half-rough interface (left) and for a fully rough interface (right). The receiver is exactly vertical to the source.

diffractions can be observed for the half-flat half-rough configuration (Fig. 5). However, the 0th and the -1 st-order diffractions are not well separated. Surprisingly, for the fully rough configuration, the \pm 1 st-order diffractions are missing and the wave packet associated to the -2 nd-order diffraction is much pronounced. This is mostly due to the symmetry of this configuration that emphasizes the destructive interferences between the \pm 1 st-order diffracted events and the constructive interferences between the \pm 2 nd-order diffractions, thus leading to an increase in the amplitude of the wave packet. These two observations are linked to the fact that a spherical wave can be seen as a superposition of several plane waves with different incident angles and thus cannot be seen if only a single plane wave is considered. The different diffraction orders generated by a plane wave incident on a periodic grating cannot interfere.

\section{Conclusion}

In this work, we have presented some results, obtained from 2D numerical simulations conducted in the time domain, on the interaction of a non-monochromatic acoustic spherical wave with a sinusoidal grating. From a selection of temporal signals that were generated, we were able to identitfy several diffraction orders. We showed that the greater the absolute value of the diffraction order, the more dispersed the associated temporal signals. Moreover, we also showed that the different signals associated to different diffraction orders can interfere and in the special case where the receiver is exactly vertical to the source the \pm 1 st-order diffractions interfere in a destructive way and therefore cancel each other. These interferences are specific to a spherical wave and cannot be observed with a single plane wave. This is a preliminary study and more works needs to be done. In this context, using of a time-domain full-wave numerical method can provide more insights into the physics of wave propagation in the presence of diffraction gratings. In particular, the influence of the size of the interface Fresnel zone, of crucial significance in the wave reflection phenomena $[19,20]$, compared to the period of the diffraction grating, needs to be investigated.

\section{Acknowledgments}

G. Gao thanks the China Scholarship Council (CSC) for the financial support. We thank the Computational Infrastructure for Geodynamics (http://geodynamics.org) which is funded by the National Science Foundation under awards EAR-0949446 and EAR-1550901. This work was granted access to the High Performance Computing resources of TGCC under allocation number A0070410305 granted by GENCI. Centre de Calcul 
Intensif d' Aix-Marseille is also acknowledged for granting access to its high performance computing resources.

\section{References}

1.F. Martini, C.J. Bean, S. Dolan, D. Marsan: Seismic image quality beneath strongly scattering structures and implications for lower crustal imaging: numerical simulations. Geophysical Journal International 145 (2001) 423-435.

2. C.J. Bean, F. Martini: Sub-basalt seismic imaging using optical-to-acoustic model building and wave equation datuming processing. Marine and Petroleum Geology 27 (2010) $555-562$

3.F. Martini, C.J. Bean: Interface scattering versus body scattering in subbasalt imaging and application of prestack wave equation datuming. Geophysics 67, 5 (2002) 1593-1601.

4. O.P. Singh Satpal, D. Sar, S.M. Chatterjee, S. Sawai: Integrated interpretation for sub-basalt imaging in Saurashtra basin, India. The Leading Edge 25, 7 (2006) 882-885.

5. M. Panzner, J.P. Morten, W.W. Weibull, B. Arntsen: Integrated seismic and electromagnetic model building applied to improve subbasalt depth imaging in the faroeshetland basin. Geophysics 81, 1 (2016) E57-E68.

6. P. Beckman, A. Spizzichino: The Scattering of Electromagnetic Waves from Rough Surfaces. Pergamon Press, Oxford, 1963.

7.F.G. Bass, I.M. Fuks: Wave Scattering from Statistically Rough Surfaces. Pergamon Press, Oxford; New York, 1979.

8. J.A. Ogilvy: Theory of Scattering from Random Rough Surfaces, Hilger, 1991.

9. G. Voronovich: Wave Scattering from Rough Surfaces. Springer, 1994.
10. W. Makinde, N. Favretto-Cristini, E. De Bazelaire: Numerical modelling of interface scattering of seismic wavefield from a random rough interface in an acoustic medium: comparison between 2D and 3D cases. Geophysical Prospecting 53, 3 (2005) 373-397.

11. E.G. Loewen, E. Popov: Diffraction Gratings and Applications. CRC Press, 1997.

12. C. Palmer: Diffraction Grating Handbook, 8th ed. MKS, 2020.

13. M. Born, E. Wolf: Principles of Optics: Electromagnetic Theory of Propagation, Interference and Diffraction of Light, 7th ed. Cambridge University Press, 1999.

14. J.W. Goodman: Introduction to Fourier optics, 4th ed. Pergamon Press, 2017.

15. S. Pinson, J. Cordioli, L. Guillon: Spherical wave reflection in layered media with rough interfaces: Three-dimensional modeling. The Journal of the Acoustical Society of America 140, 2 (2016) 1108-1115.

16. J. Tromp, D. Komatitsch, Q. Liu: Spectral-element and adjoint methods in seismology. Communications in Computational Physics 3, 1 (2008) 1-32.

17. Z. Xie, R. Matzen, P. Cristini, D. Komatitsch, R. Martin: A new PML formulation for coupled fuid-solid problems: Application to numerical simulations in ocean acoustics with solid ocean bottoms. Journal of the Acoustical Society of America 140 (2016) 165.

18. F. Auger, P. Flandrin: Improving the readability of timefrequency and time-scale representations by the reassignment method. IEEE Transactions on Signal Processing 43, 5 (May 1995) 1068-1089.

19. N. Favretto-Cristini, P. Cristini, E. de Bazelaire: What is a seismic reflector like? Geophysics 74, 1 (2009) T13-T23.

20. N. Favretto-Cristini, P. Cristini, E. de Bazelaire: Influence on the Interface Fresnel zone on the reflected P-wave amplitude modelling. Geophysical Journal International 171 (2007) 841-846.

Cite this article as: Gao G, Cristini P, Favretto-Cristini N \& Deumié C. 2021. On the reflection of time-domain acoustic spherical waves by a sinusoidal diffraction grating. Acta Acustica, $\mathbf{5}, 6$. 